\title{
Annotation and Classification of Graphs of Property Values Reported in Material Science Literature
}

\section{Naoki linuma ( $\nabla$ sd20403@toyota-ti.ac.jp )}

Toyota Technological Institute https://orcid.org/0000-0002-9095-390X

\section{Fusataka Kuniyoshi}

National Institute of Advanced Industrial Science and Technology: Kokuritsu Kenkyu Kaihatsu Hojin Sangyo Gijutsu Sogo Kenkyujo https://orcid.org/0000-0001-5914-009X

\section{Jun Ozawa}

National Institute of Advanced Industrial Science and Technology: Kokuritsu Kenkyu Kaihatsu Hojin Sangyo Gijutsu Sogo Kenkyujo https://orcid.org/0000-0003-4212-9008

\section{Makoto Miwa}

Toyota Technological Institute: Toyota Kogyo Daigaku https://orcid.org/0000-0002-2330-6972

\section{Research article}

Keywords: Annotation, Figure classification, Materials Informatics, Multimodal information, Information Extraction

Posted Date: June 30th, 2021

DOl: https://doi.org/10.21203/rs.3.rs-637136/v1

License: (c) (1) This work is licensed under a Creative Commons Attribution 4.0 International License. Read Full License 
Annotation and Classification of Graphs of Property Values Reported in Material Science Literature

Naoki linuma ${ }^{1,2^{*}}$, Kuniyoshi Fusataka ${ }^{1,3}$, Jun Ozawa ${ }^{1,3}$ and Makoto Miwa ${ }^{1,2} \quad 9$

10 
${ }^{1}$ Introduction

${ }^{2}$ Given the emergence of data science and machine learning in materials science, ${ }^{2}$

${ }^{3}$ increased importance is placed on obtaining data. Data in materials science are ${ }^{3}$

${ }^{4}$ particularly heterogeneous due to the significant wide range of classes of materials ${ }^{4}$

${ }^{5}$ and the variety of material properties. In recent studies, several extraction tools ${ }^{5}$

${ }^{6}$ have been applied, based on natural language processing from literature $[1,2,3]{ }^{6}$

${ }^{7}$ However, these data may appear in several ways in literature, such as tables and fig- ${ }^{7}$

${ }^{8}$ ures, which are often ignored in natural language processing, although they contain ${ }^{8}$

${ }^{9}$ a significant amount of data on experimental measurements of materials proper- ${ }^{9}$

${ }^{10}$ ties. In this study, we focus particularly on graphs, which are figures representing ${ }^{10}$

${ }^{11}$ graphically numerical data, such as a bar graph and line graph, because they are of- ${ }^{11}$

${ }^{12}$ ten employed to show the evidence supporting the main claims in materials science ${ }^{12}$

${ }^{13}$ literature and thus often include the most important information in the literature. ${ }^{13}$

14 Several methods have recently been reported to automatically consume and codify ${ }^{14}$

${ }^{15}$ information in figures of scientific literature across domains, such as image classi- ${ }^{15}$

${ }^{16}$ fication and optical character recognition $[4,5]$, based on techniques adapted from ${ }^{16}$

${ }^{17}$ the field of computer vision. These methods have immense potential to obtain data ${ }^{17}$

${ }^{18}$ necessary for data-driven materials research based on the literature. Furthermore, ${ }^{18}$

${ }^{19}$ there have been several studies on identifying the characteristic values from fig- ${ }^{19}$

${ }^{20}$ ures and tables in literature using machine-learning-based approaches; for example, ${ }^{20}$

${ }^{21}$ parsing result figures [6], extracting the values from tables [7], classifying the fig- ${ }^{21}$

${ }^{22}$ ures of biomedical articles on five predefined figure types [8], and quantifying data ${ }^{22}$

${ }^{23}$ from microscopy images [9]. However, in the field of materials science, there is no ${ }^{23}$

${ }^{24}$ reported general method for identifying the characteristic values of graphs. $\quad 24$

${ }^{25}$ In this study, as the first step towards automatic information extraction from ${ }^{25}$

${ }^{26}$ graphs, we focus on automatically extracting and classifying graphs in the materials ${ }^{26}$

${ }^{27}$ science literature. To achieve this, we construct a dataset that classifies graphs ${ }^{27}$

${ }^{28}$ according to types of conditions. We define new conditions for graph classification ${ }^{28}$

${ }^{29}$ based on the examination of actual graphs reported in the "Journal of Material ${ }^{29}$

${ }^{30}$ Chemistry A (2015-2019)"[1] in this paper (see Figures 3 and 4). We annotated the ${ }^{30}$

${ }^{31}$ types based on the images and captions. Furthermore, we propose deep-learning- ${ }^{31}$

${ }^{32}$ based classification models that utilize multimodal information of graphs, consisting ${ }^{32}$

${ }^{33}{ }_{[1]}$ https://www.rsc.org/journals-books-databases/about-journals/journal-of-materials-chemistry-a/ ${ }^{33}$ 
${ }^{1}$ of graph images, text in graphs, and captions. A representative process for the ${ }^{1}$ ${ }^{2}$ classification of graphs is shown in Fig. 1. We first prepare baseline models for ${ }^{2}$ ${ }^{3}$ each modal information, which are popularly employed as baselines for natural ${ }^{3}$ ${ }^{4}$ language processing and image processing. We then consider two models to combine ${ }^{4}$ ${ }^{5}$ the unimodal information: one integrates the feature representations of unimodal ${ }^{5}$ ${ }^{6}$ models, while the other aggregates the prediction results of unimodal models. The ${ }^{6}$ ${ }^{7}$ best multimodal model classified graphs with a micro-F1 score of $96.1 \%$ using the ${ }^{7}$ ${ }^{8}$ proposed dataset, which represents a better performance than unimodal models. ${ }^{8}$

9 The primary contributions of this study can be summarized as follows: 9

$10 \quad 10$

- We construct a dataset classifying graphs reported in the materials science literature using pre-defined conditions.

12

- The experimental results show that the constructed dataset can be used to train a deep-learning-based model, which classifies graphs according to their characteristic conditions with a micro-F1 score of $96.1 \%$. This demonstrates that the dataset is useful for training the deep-learning-based model.

16

- We show that using the multimodal information of graphs can improve the classification performance. ${ }_{22} \mathrm{Our}$ dataset is a set of triples of graph images, captions, and labels, obtained based22 23on property conditions. The overview of the construction of the property valuezs 24graph dataset from published papers is shown in Fig. 2. The dataset is constructed24 25by extracting graph images from a large collection of published journal papers in25 26the field of materials science. Then, we label the images leveraging crowdsourcing,26 27enabling the creation of large datasets in short periods. In the following sections,27 28we explain the construction in detail. 28

${ }^{31}$ To classify the images according to their corresponding conditions, we define the ${ }^{31}$ ${ }^{32}$ types of graphs using the following conditions. Examples of each type and graph ${ }^{32}$ 33 are shown in Figures 3 and 4. 
1 Temperature: The temperature condition is a parameter for heat treatment, which ${ }^{1}$

2 is a process of heating and cooling a material to improve its properties $\left(\mathrm{see}^{2}\right.$ Figure 3(a)).

4 Time: The time condition is a parameter for the time course of each process and ${ }^{4}$

5 measurement in materials synthesis (see Figure 3(b)).

${ }^{6}$ Angle: The angle condition is a parameter for analyzing the X-ray diffraction ${ }^{6}$

$7 \quad$ patterns to determine the state and physical properties of materials (see Figure ${ }^{7}$

8 $3(\mathrm{c}))$.

8

${ }^{9}$ Wavelength: The wavelength condition is a parameter often used to analyze the ${ }^{9}$ components of materials (see Figure $3(\mathrm{~d})$ ) by various methods, such as ab- ${ }^{10}$ sorption spectroscopy.

Capacity: The capacity condition is a parameter related to battery performance. ${ }^{12}$ To represent the performance, charge and discharge curves are often drawn ${ }^{13}$ (Figure 3(f)).

${ }^{15}$ Pressure: The pressure condition is a parameter related to the absorption and ${ }^{15}$ desorption operations on the surfaces of materials. For example, it is shown ${ }^{16}$ on the horizontal axes of adsorption-desorption isotherms (see Figure $3(\mathrm{~g})$ ). ${ }^{17}$

Ohm: The ohm condition is a parameter related to the performance of materials as ${ }^{18}$ counter electrodes. To analyze it, Nyquist plots are drawn many times (Figure ${ }^{19}$ $3(\mathrm{~g}))$.

Voltage: The voltage condition is a parameter that reveals the electrochemical ${ }^{21}$ reaction mechanism of materials. For this purpose, discharge and charge curves ${ }^{22}$ are drawn (Figure $3(\mathrm{~h})$ ).

Energy: The energy condition is used in several methods for the analysis of the ${ }^{24}$ compositions of materials, such as X-ray spectroscopy and X-ray Absorption ${ }^{25}$ Near Edge Structure ( Figure 3(i)).

Raman shift: The Raman shift condition is a parameter used in Raman spec- ${ }^{27}$ troscopy, which measures Raman intensity to evaluate the physical properties ${ }^{28}$ of materials (Figure $3(\mathrm{j})$ ).

Cycle: The cycle condition is a parameter used for the evaluation of the material ${ }^{30}$ durability against various indices (Figure $4(\mathrm{k})$ ).

Other: Graphs of measurement conditions other than the 11 listed above (Figure ${ }^{32}$ $4(1))$. 
1 Additionally, we define a label "Not target" in classifying the figures reported in ${ }^{1}$

${ }^{2}$ the paper, which indicates figures that are not graphs. Such figures include pho- ${ }^{2}$

${ }^{3}$ tographs, images of compounds, and diagrams with multiple figures, as shown in ${ }^{3}$

${ }^{4}$ Fig. 4.

${ }_{6}$ Collecting paper data

${ }_{7}$ The journal named "Journal of Material Chemistry A (2015-2019)"[2] from the ${ }_{8}$ Royal Society of Chemistry (RSC), which mainly publishes papers dealing with the ${ }_{8}$

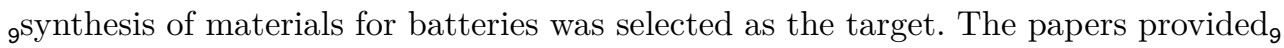
${ }_{10}$ by RSC are in the XML format and contain figures, tables, and schemes. $\mathrm{The}_{10}$ ${ }_{11}$ statistics of the target article data are listed in Table 1.

${ }_{17}$ As shown in Fig. 5, a figure extracted from the paper may contain a single $\operatorname{graph}_{17}$ ${ }_{18}$ or multiple graphs, referred to as "one-graph figure" or "multiple-graph figure," 18 ${ }_{19}$ respectively.

We aim to construct a dataset containing only one-graph figures to avoid any difods. One-graph figures were extracted using the Compound Figure Separator (CFS) proposed by Tsutsui et al. [10]. The figures that were classified as 0 or 1 figure by CFS were chosen as one-graph figures. We extracted 8,634 one-graph figures from 28 91,019 figures obtained from 14,071 papers, as shown in Table 1. Multiple-graph 29 figures were extracted by applying regular expressions to the figure captions. This 30 rule judges whether a caption contains "(a-z)" or "(A-Z)" and extracts the cor31 responding figures as multiple-graph figures. For example, if we apply the rule to 32 the caption "(a) X-ray diffraction patterns of samples; SEM images of (b) carbon

${ }^{33}{ }_{[2]}$ https://www.rsc.org/journals-books-databases/about-journals/journal-of-materials-chemistry-a/ ${ }^{33}$ 
${ }^{1}$ spheres, (c and d) $\mathrm{MnO}_{2}$, and (e and f) $\mathrm{MnO}_{2} / \mathrm{C}$ composite" (Figure 1 of [11]), the ${ }^{1}$

${ }^{2}$ figure is extracted as a multiple-graph figure because it contains four instances of ${ }^{2}$ 3"(a-z)" or "(A-Z)" in the caption. As a result, 64,361 multiple-graph figures were ${ }^{3}$ ${ }^{4}$ extracted.

5 Next, we designed a binary classification model for one-graph and multiple-graph ${ }^{5}$ ${ }^{6}$ figures using Lobe ${ }^{[3]}$, which is a machine learning tool for easily training the custom ${ }^{6}$ ${ }^{7}$ image classifier. We randomly sampled 1,000 one-graph images and 1,000 multiple- ${ }^{7}$ ${ }^{8}$ graph images to train the classifier. We applied the trained classifier using Lobe to ${ }^{8}$ ${ }^{9}$ the 91,091 images from 14,071 papers and extracted 16,668 one-graph figures. 10

11 Annotation details

12Annotation was performed using crowdsourcing to reduce the time required. We12 ${ }^{13}$ employed annotators via the Amazon Mechanical Turk ${ }^{[4]}$ to label the extracted ${ }^{13}$ ${ }^{14}$ graph images. To ensure the quality of the dataset, a maximum of nine annotators ${ }^{14}$ ${ }^{15}$ were used to annotate one figure. The annotation process took about three days, ${ }^{15}$ 16and 732 annotators participated in this task. 16 17 17 ${ }_{18}$ Label integration ${ }_{19}$ Our dataset contains up to nine labels per figure, but there are cases with variations ${ }_{19}$ ${ }_{20}$ in labels due to the complexity of the figure, misunderstandings, etc. Therefore, 20 ${ }_{21}$ we implemented majority voting, which is a typical quality management $\operatorname{method}_{21}$ ${ }_{22}$ for crowdsourcing. Majority voting is implemented by selecting the label with the ${ }_{22}$ ${ }_{23}$ highest number of occurrences among the annotated labels. In addition, we set $\mathrm{a}_{23}$ ${ }_{24}{ }_{\text {threshold on the agreement rate in the majority vote and extracted only annotation }}{ }_{24}$ ${ }_{25}$ data with agreement rates above the threshold. To determine the threshold, $100_{25}$ ${ }_{26}$ figures were annotated by two annotators. The agreement between the two annota- 26 ${ }_{27}$ tors was $83 \%$. Considering that the number of annotators is more than two, we $\operatorname{set}_{27}$ ${ }_{28}$ the threshold to $80 \%$ Table 2 shows the size of the dataset after label integration. 28 ${ }_{32}$ an annotator. We evaluated the annotations by comparing them with the labels of ${ }_{32}$ $33^{[3]}$ https://www.lobe.ai/

${ }^{33}{ }^{[4]}$ https : //www.mturk.com/ 
1

5

8

10

11

12

${ }^{13}$ the dataset. The results show that the accuracy of the labels in the dataset was ${ }^{14} 99 \%[5]$, indicating that the labeling is accurate.

15

16 17

Classification with unimodal information

${ }_{18}$ We utilize three types of information: graph images, graph text extracted using $_{18}$ ${ }_{19}$ Textract $^{[6]}$, and captions for classification. In this section, we discuss classification ${ }_{19}$ ${ }_{20}$ using each of the three types of information. We prepared different classification ${ }_{20}$ ${ }_{21}$ models for each type of information (unimodal information). In the following sec- $_{21}$ ${ }_{22}$ tions, we describe graph image classification, graph text classification, and caption ${ }_{22}$ ${ }_{23}$ classification in order.

24

${ }^{25}$ Graph image classification

${ }^{26}$ For image-based classification, we use two models, viz. ResNet[12] and EfficientNet[13], ${ }^{27}$ which have been reported to perform well in image-based tasks and can easily em- ${ }^{27}$ ${ }^{28}$ ploy transfer learning. We trained these models by fine-tuning them on the con- ${ }^{28}$ ${ }^{29}$ structed dataset.

30

${ }_{31}{ }^{[5]}$ Please note that the 100 figures sampled here are different from those in the last ${ }_{32}$ section. The figures in the last section are noisy. They include difficult cases that ${ }_{32}$ ${ }_{33}$ cause disagreement among annotators in crowd sourcing ${ }^{33}{ }^{[6]}$ https : //aws . amazon. com/textract/
10

11

12

14 15 16 17 
1 ResNet is a model that can learn deep layered models by introducing residual ${ }^{1}$

${ }^{2}$ connections. In our experiment, the images were resized to $224 \times 224$ pixels, and ${ }^{2}$

${ }^{3}$ we employed ResNet50. 3

4 EfficientNet is a model that achieves high performance with fewer parameters ${ }^{4}$

${ }^{5}$ than conventional models through model scaling. EfficientNet-B0 was used in our ${ }^{5}$

6 experiments for the same reason as ResNet.

Here, we describe the flow of prediction using these two models.

First, we create a feature vector $\hat{\boldsymbol{x}}$ from the input image tensor $\boldsymbol{x}$ using these two

models. Since each input image is an RGB image resized to $224 \times 224$ pixels, the

10

11

dimensions of the input image tensor $\boldsymbol{x}$ are $(224,224,3)$.

12

13

$$
\hat{\boldsymbol{x}}=\operatorname{Model}(\boldsymbol{x})
$$

14

Please note that the dimensions of the feature vector $\hat{\boldsymbol{x}}$ are different for these two 15 models.

16

The feature vector $\hat{\boldsymbol{x}}$ is then passed to a single-layer fully connected (FC) network, ${ }_{17}$ ${ }_{18}$ and finally, the probability $\boldsymbol{p}$ of each label is calculated by applying a softmax ${ }_{18}$ function for prediction.

20

21

22

$\boldsymbol{p}(\boldsymbol{y} \mid \boldsymbol{x} ; \boldsymbol{\theta})=\operatorname{softmax}(\boldsymbol{o})$

23

24 Here, $\boldsymbol{\theta}$ represents the parameters of the model, and $\boldsymbol{y}$ represents a set of candidate ${ }_{24}$ ${ }_{25}$ labels for prediction. We choose the label with the highest probability $\boldsymbol{p}$ for the input ${ }_{25}$ ${ }_{26}$ sentence representation $\boldsymbol{x}$ as the prediction result.

${ }^{29}$ In this section, we explain the flow of prediction using text extracted by Textract ${ }^{29}$

${ }^{30}$ from the graph images. 30

31 Since Textract extracts text word by word without any contextual information, ${ }^{31}$

${ }^{32}$ the feature vector of the text $\boldsymbol{t}$ is represented by a bag-of-words (BoW) or a term ${ }^{32}$

${ }^{33}$ frequency-inverse document frequency (TF-IDF). 
1 The probability $\boldsymbol{p}$ of each label is predicted by passing the feature vector $\boldsymbol{t}$ through $^{1}$

${ }^{2}$ a two-layer fully connected network and applying a softmax function to the output. ${ }^{2}$

3

8 Here, $R e L U$ is the nonlinear activation function

9

10 Caption classification

$$
\hat{\boldsymbol{t}}=F C_{1}(\boldsymbol{t})
$$$$
\boldsymbol{o}=F C_{2}(\operatorname{Re} L U(\hat{\boldsymbol{t}}))
$$$$
\boldsymbol{p}(\boldsymbol{y} \mid \boldsymbol{x} ; \boldsymbol{\theta})=\operatorname{softmax}(\boldsymbol{o})
$$

Two models are used for classification with caption texts: a convolutional neural 11 network $(\mathrm{CNN})$ as a baseline, and a CNN with Mat-Word2Vec [14] (Mat-CNN), which is domain-specific word embedding for materials science.

13

The CNN creates feature representations of sentences from randomly initialized 14 word representations and predicts the labels based on the representations. In the 15 following paragraphs, we explain the prediction process. 16 17 First, we obtain the representation of an input sentence $s$ using random word representations.

19

$$
\boldsymbol{s}=\left[\boldsymbol{w}_{0}^{T}, \boldsymbol{w}_{1}^{T}, \ldots, \boldsymbol{w}_{N-1}^{T}\right]
$$

21 where $\boldsymbol{s}$ is the list of 100 -dimensional vector representations $\boldsymbol{w}$ of words, and $N$ is21 22the maximum sentence length.

23 Next, we generate a feature vector of the sentence $\hat{\boldsymbol{s}}$ using the CNN from the word23 24representations $s$.

$$
\hat{s}=C N N(s)
$$

${ }^{27}$ Finally, as shown in Eqs. (2) and (3), the feature vector $s$ is passed through a ${ }^{27}$ ${ }^{28}$ single-layer FC neural network, and the probability $\boldsymbol{p}$ of each label is calculated by ${ }^{28}$ ${ }^{29}$ applying a softmax function to the output. We choose the label with the highest ${ }^{29}$

30 o 30 probability $\boldsymbol{p}$ for the input sentence representation $s$ as the prediction result.

${ }^{31}$ For Mat-CNN, we initialize the word representation $\boldsymbol{w}$ of Materials Science Word ${ }^{31}$ ${ }^{32}$ Embeddings, acquired by Kim et al. from literature using Word2Vec [14]. We pre- ${ }^{32}$

33 dicted the relation in the same manner as for the CNN described above. 33 dicted the relation in the same manner as for the CNN described above. 
${ }^{1}$ Classification with two types of multimodal information $\quad 1$

${ }^{2}$ We consider the prediction based on any two out of the following three types of

3. information: graph image, graph text, and captions.

4 5 The feature representation vectors of two types of information are combined to ${ }_{6}$ produce the final feature representation vector $\boldsymbol{h}_{1}$. For instance, to create a feature representation vector from an image and its caption, we combine their representa7 tions, as shown in Eqs. (1) and (8): $\boldsymbol{h}_{1}=[\boldsymbol{x} ; \hat{\boldsymbol{s}}]$. ${ }_{9}$ Finally, we pass the feature vector $\boldsymbol{h}_{1}$ through a single-layer FC neural network, 9 ${ }_{10}$ according to Eqs. (2) and (3), and apply a softmax function to the output to predict ${ }_{10}$ ${ }_{11}$ the probability $\boldsymbol{p}$ of each label.

12

${ }^{13}$ Classification with multimodal information 13

${ }^{14}$ We adopt two approaches to combine the three types of information: one involves ${ }^{14}$ ${ }^{15}$ combining features similar to the case of two multimodal models, and the other ${ }^{15}$ ${ }^{16}$ involves ensembling each type of model. 16 $17 \quad 17$ The method for combining the features is the same as that for using two types 18 of information. Using Eqs. (1), (4) and (8), the feature vector for prediction is 19 represented as $\boldsymbol{h}_{1}=[\boldsymbol{x} ; \hat{\boldsymbol{t}} ; \hat{\boldsymbol{s}}]$. We refer to this method as the Concat method.

20 In the second method, predictions are made by multiple models trained separately ${ }_{22}$ on each type of information, and the final prediction label is determined by their ${ }_{22}$ ${ }_{23}$ majority vote. We refer to this method as the Ensemble method.

24

25Implementation Details 25

${ }^{26}$ We used a g4dn.4xlarge instance of Amazon Elastic Compute Cloud (Amazon ${ }^{26}$ $\left.{ }^{27} \mathrm{EC} 2\right)^{[7]}$ as the computing environment. The g4dn.4xlarge instance contains Second- ${ }^{27}$ ${ }^{28}$ Generation Intel Xeon Scalable (Cascade Lake) processors and an NVIDIA T4 Ten- ${ }^{28}$ ${ }^{29}$ sor Core GPU. We used the machine learning library PyTorch ${ }^{[8]}$ to implement the ${ }^{29}$ ${ }^{30}$ model described above. 31 31 $32^{[7] \text { https://aws. amazon.com/ec2/?ec2-whats-new. sort-by=item. additionalFields. postDateTime\& }} 32$

${ }^{33}{ }_{[8]}^{\text {ec2-whats }}$ https://pytorch.org/ 
2

5
Table 3: Search range for hyper-parameters

\begin{tabular}{l|c}
\hline \hline Optimizer & Adam[17], Momentum SGD [18] \\
\hline Learning rate & $1 e^{-4}-1 e^{-2}$ \\
\hline Weight decay & $1 e^{-10}-1 e^{-3}$ \\
\hline Batch size & {$[16,32]$} \\
\hline Dropout rate & $0.0-0.5$ \\
\hline Convolution filter size & {$[3,5,7]$} \\
\hline Number of filters & {$[50,100,200]$} \\
\hline \hline
\end{tabular}

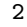

3

6

7

8

${ }^{8}$ Results and discussion

${ }^{9}$ In this section, we evaluate the performance of the trained graph classifier using the ${ }^{9}$

${ }^{10}$ constructed dataset.

11

12Experimental setup

13In this section, we explain the evaluation method and the setting of various hyper-13

14parameters for evaluation.

14

15

15

${ }_{16}$ Evaluation method

16

${ }_{17}$ We employed the holdout method to evaluate the classification performance and $_{17}$ ${ }_{18}$ the F-score was used as an evaluation index. The dataset was divided into train- ${ }_{18}$ ${ }_{19}$ ing, development, and test datasets in a ratio of $6: 2: 2$, such that the labels were ${ }_{19}$ ${ }_{20}$ distributed close to each other.

20

21

Setting various hyperparameters

22

The hyper-parameters during training were determined by choosing them from 23

the parameters presented in Table 3 . We aimed at producing the highest micro24 averaged F-scores on the development dataset. Tuning was performed by pruning 25 the branches with the Successive Halving [15] algorithm, implemented in a hyper26 parameter optimization framework Optuna[16]. During tuning, early stopping was 27 performed at 20 epochs.

${ }^{29}$ Craph image classification 29

${ }^{30}$ We trained and evaluated EfficientNet and ResNet for graph image classification; ${ }^{30}$

${ }^{31}$ the results are listed in Table 4. EfficientNet showed the higher performance be- ${ }^{31}$

${ }^{32}$ tween the two models for all labels, while the labels of "Not target" and "Voltage"

${ }^{33}$ are low. This suggested that it is difficult to extract the features of "Not target" 
2

5

6

7

8

10

11

12

13

${ }^{14}$ and "Voltage" from the images, and the images of "Not target" are considered to ${ }^{14}$ ${ }^{15}$ contain other diagrams (e.g., microscopic images, flowcharts, and other diagrams ${ }^{15}$ ${ }^{16}$ with diverse variations), which may increase the difficulty of classification. The ${ }^{16}$ ${ }^{17}$ images of "Voltage" were also considered more difficult to classify than the other ${ }^{17}$ ${ }^{18}$ images because they are similar to some images of "Energy". The micro-averaged ${ }^{18}$ ${ }^{19}$ and macro-averaged $\mathrm{F}$-scores for each label did not differ significantly among the ${ }^{19}$ ${ }^{20}$ models. However, the F-score for each label varied, indicating that each model was ${ }^{20}$ ${ }^{21}$ accurate for different labels.

22 In addition, to check the tendency of misclassification in the graph images, we ${ }^{22}$ ${ }^{23}$ employ the confusion matrix of EfficientNet, which shows the highest classification ${ }^{23}$ ${ }^{24}$ performance (Figure 6). The vertical axis shows the correct labels and the horizontal ${ }^{24}$ ${ }^{25}$ axis shows the labels predicted by the model. Figure 6 shows that there were many ${ }^{25}$ ${ }^{26}$ errors in "Other" classification. In particular, there are many cases where "Other" is ${ }^{26}$ ${ }^{27}$ mistakenly predicted as "Capacity" or "Cycle" and "Time" is mistakenly predicted ${ }^{27}$ ${ }^{28}$ as "Other". "Other" contains various graphs that do not belong to the labels defined ${ }^{28}$ ${ }^{29}$ according to the material properties. Therefore, We consider that graphs that are ${ }^{29}$ ${ }^{30}$ similar in general shape with "Capacity", "Cycle", and "Time" are also included ${ }^{30}$ ${ }^{31}$ in "Other", which may have caused the error. Such error cases suggest that it is ${ }^{31}$ ${ }^{32}$ very difficult to classify information related to the context of the graph, such as ${ }^{32}$ 33

properties, based on the general shape of the graph using images.

\section{2}

(1)
(1) s ( 7 8

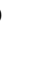
10 
1

2

5

8

10

11

12

13

${ }_{14}$ Graph text classification
Table 5: Graph text classification

\begin{tabular}{c|ccc}
\hline & BoW & TF-IDF & 2 \\
\hline \hline Temperature & 0.948 & 0.922 & 3 \\
Time & 0.948 & 0.923 & 4 \\
Angle & 0.940 & 0.935 & \\
Wavelength & 0.990 & 0.945 & 5 \\
Capacity & 0.800 & 0.737 & 6 \\
Pressure & 0.980 & 0.948 & \\
Ohm & 0.829 & 0.817 & \\
Voltage & 0.855 & 0.748 & \\
Energy & 0.882 & 0.857 & \\
Raman shift & 0.985 & 0.970 & 1 \\
Cycle & 0.826 & 0.826 & 11 \\
Other & 0.543 & 0.475 & 12 \\
Not target & 0.940 & 0.893 & \\
\hline Macro & 0.882 & 0.846 & \\
Micro & 0.928 & 0.894 &
\end{tabular}

${ }_{15}$ The text extracted by Textract was classified by creating a feature representation ${ }_{15}$

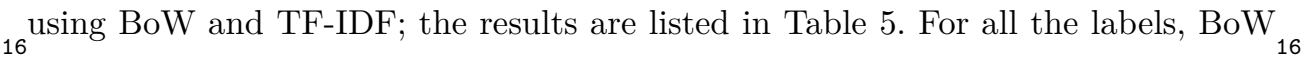
${ }_{17}$ produced higher F-scores than TF-IDF.

${ }_{18}$ Similar to image classification, we show the confusion matrix of BoW, which has ${ }_{18}$ ${ }_{19}$ the highest classification performance, in Figure 7. There were many errors in the ${ }_{19}$ 20 "Other" class. Particularly, there were many cases where "Other" was misclassi- ${ }_{20}$ ${ }_{21}$ fied as "Angle" or "Not target". Many of the images of "Angle" have intensity on ${ }_{21}$ ${ }_{22}$ the vertical axes. On the other hand, the images of "Other" occasionally have the ${ }_{22}$ ${ }_{23}$ intensity as that in the vertical axis. Therefore, we consider that the BoW of the ${ }_{23}$ ${ }_{24}$ "Other" sometimes resembles that of the "Angle", causing misclassification between ${ }_{24}$ ${ }_{25}$ them. We believe that "Other" is mistaken as "Not target" because it is difficult ${ }_{25}$ ${ }_{26}$ to create features for both. In addition, there are many cases where "Voltage" was ${ }_{26}$ ${ }_{27}$ misclassified as "Not target". The images of "Voltage" occasionally have some text ${ }_{27}$ ${ }_{28}$ in images of figures. We consider that the model misclassified "Voltage" as "Not ${ }_{28}$ ${ }_{29}$ target" because there are many images containing little or no text in "Not target", ${ }_{30}$ such as microscopic images and flowcharts.

${ }^{31}$ Caption classification

30

${ }^{32}$ We trained and evaluated $\mathrm{CNN}$ and Mat-CNN using only the figure captions; the ${ }^{32}$ ${ }^{33}$ results are shown in Table 6, with Mat-CNN showing the best performance. For all ${ }^{33}$ 
1

2

5

6

7

8

9

10

11

12

13

Table 6: Classification using captions

\begin{tabular}{c|ccc}
\hline & CNN & Mat-CNN & 2 \\
\hline \hline Temperature & 0.792 & 0.816 & 3 \\
Time & 0.707 & 0.769 & 4 \\
Angle & 0.944 & 0.955 & 5 \\
Wavelength & 0.899 & 0.901 & \\
Capacity & 0.167 & 0.167 & 6 \\
Pressure & 0.800 & 0.839 & 7 \\
Ohm & 0.961 & 0.950 & \\
Voltage & 0.738 & 0.803 & \\
Energy & 0.744 & 0.763 & 1 \\
Raman shift & 0.853 & 0.811 & 1 \\
Cycle & 0.660 & 0.726 & \\
Other & 0.000 & 0.207 & 0.928 \\
Not target & 0.870 & 0.741 & 0.851 \\
\hline Macro & 0.703 & &
\end{tabular}

${ }_{15}$ than $\mathrm{CNN}$, indicating that the word representations pre-trained from literature are ${ }_{15}$ ${ }_{16}$ useful for figure classification.

${ }_{17}$ We show the confusion matrix of Mat-CNN in Figure 8. Several errors were ob- ${ }_{17}$ ${ }_{18}$ served for "Time". Particularly, there were many cases in which time was misclas- ${ }_{18}$ ${ }_{19}$ sified as "Cycle", "Not target", "Other", or "Voltage". In several cases, the model ${ }_{19}$ ${ }_{20}$ also predicted "Time" erroneously. In particular, there were cases in which "Cycle", ${ }_{21}$ "Not target", "Other", or "Voltage" were misclassified as "Time". The distribution ${ }_{21}$ ${ }_{22}$ of labels that are easily mispredicted as "Time" and those that are easily mispre${ }_{23}$ dicted by "Time" are similar, suggesting that the contextual feature representation ${ }_{23}$ ${ }_{24}$ of "Time"'s captions is similar to that of misclassified labels.

${ }^{25}$ Classification with two multimodal information

24

${ }^{26}$ When considering two types of information, we used the model that showed the best ${ }^{26}$ ${ }^{27}$ performance for individual training. Specifically, the EfficientNet, BoW, and Mat- ${ }^{27}$ ${ }^{28} \mathrm{CNN}$ models were used for the image of the figure, the text extracted by Textract ${ }^{28}$ ${ }^{29}$ from the images, and the captions, respectively.

30 The results are shown in Table 7. Image+Text and Text+Caption showed similar ${ }^{30}$ ${ }^{31}$ overall performances, but Text+Caption showed slightly higher micro-averaged and ${ }^{31}$ ${ }^{32}$ macro-averaged F-scores for each label. Furthermore, the F-score of Caption+Image ${ }^{32}$ ${ }^{33}$ was much lower than those of Image+Text and Text+Caption, indicating that the ${ }^{33}$ 


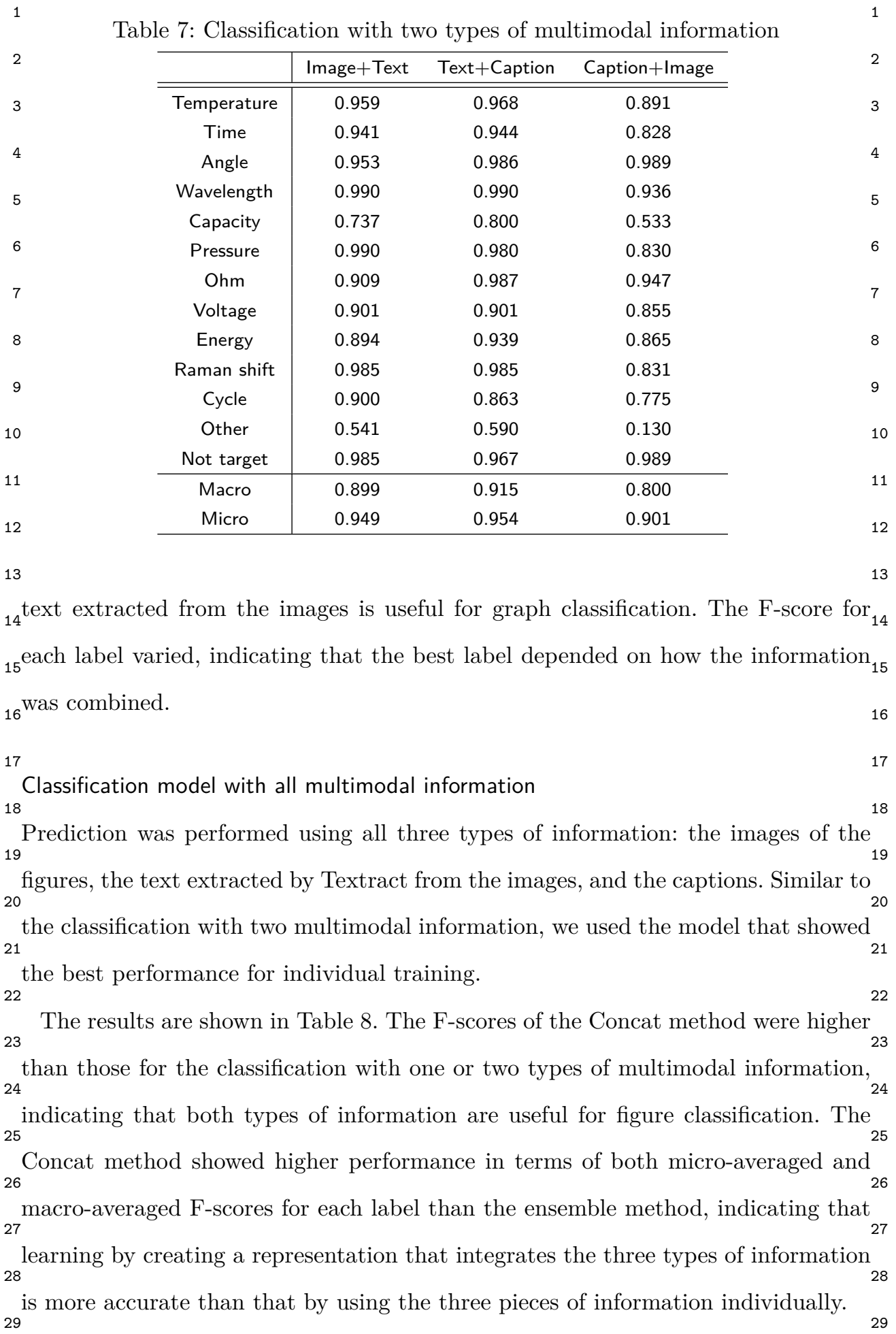

\section{${ }^{30}$ Conclusions $\quad{ }^{30}$}

${ }^{31}$ In this paper, we proposed an approach to classify graphs according to their property ${ }^{31}$

${ }^{32}$ conditions. We constructed a manually annotated dataset for classification using ${ }^{32}$

${ }^{33}$ property conditions and evaluated the same. We also proposed and evaluated deep- ${ }^{33}$ 
5

8

10

11

12

13

14learning-based classification models in both unimodal and multimodal settings. To14

15the best of our knowledge, this is the first study to classify graphs according to their15

${ }^{16}$ property conditions using multimodal information with deep learning models. $\quad 16$

17 The results showed that the models label the graphs and classify property condi- ${ }^{17}$

${ }^{18}$ tions, with micro-averaged F-score as high as 0.961 . Furthermore, we showed that ${ }^{18}$

${ }^{19}$ the simultaneous use of the graph images, the text in the graphs, and the captions ${ }^{19}$

${ }^{20}$ can improve the classification performance.

${ }^{21}$ In future, we will improve these classification models. We consider several ways to ${ }^{21}$

22.22 improve the performance of these models. First, we will investigate the method to

23 . 23 achieve more effective use of multimodal information. Second, we will consider incor-

${ }^{24}$ porating the in-text citations of figures in the manuscript. Third, we will construct ${ }^{24}$

${ }^{25}$ a model that can handle multiple-graph figures. We will also consider the automatic ${ }^{25}$

26

extraction of information from graphs and other non-textual components. 26 27

28

29Abbreviations

RSC: Royal Society of Chemistry

${ }^{30}$ CFS: Compound Figure Separator

${ }_{31}$ FC: fully connected

BoW: bag-of-words

32TF-IDF: term frequency-inverse document frequency

${ }_{33} \mathrm{CNN}$ : convolutional neural network

Mat-CNN: convolutional neural network with Mat-Word2Vec
1

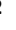
3 4 5 6 7 8

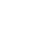
10 11 12 13

\begin{tabular}{c|ccc}
\hline & Concat & Ensemble & 2 \\
\hline \hline Temperature & 0.962 & 0.952 & 3 \\
Time & 0.959 & 0.929 & 4 \\
Angle & 0.997 & 0.990 & 5 \\
Wavelength & 0.993 & 0.988 & 5 \\
Capacity & 0.700 & 0.625 & 6 \\
Pressure & 0.970 & 0.879 & \\
Ohm & 0.974 & 1.000 & 7 \\
Voltage & 0.940 & 0.944 & 8 \\
Energy & 0.918 & 0.957 & \\
Raman shift & 0.985 & 0.971 & 9 \\
Cycle & 0.907 & 0.916 & 10 \\
Other & 0.603 & 0.415 & 11 \\
Not target & 0.981 & 0.969 & 0.887
\end{tabular}

4

25




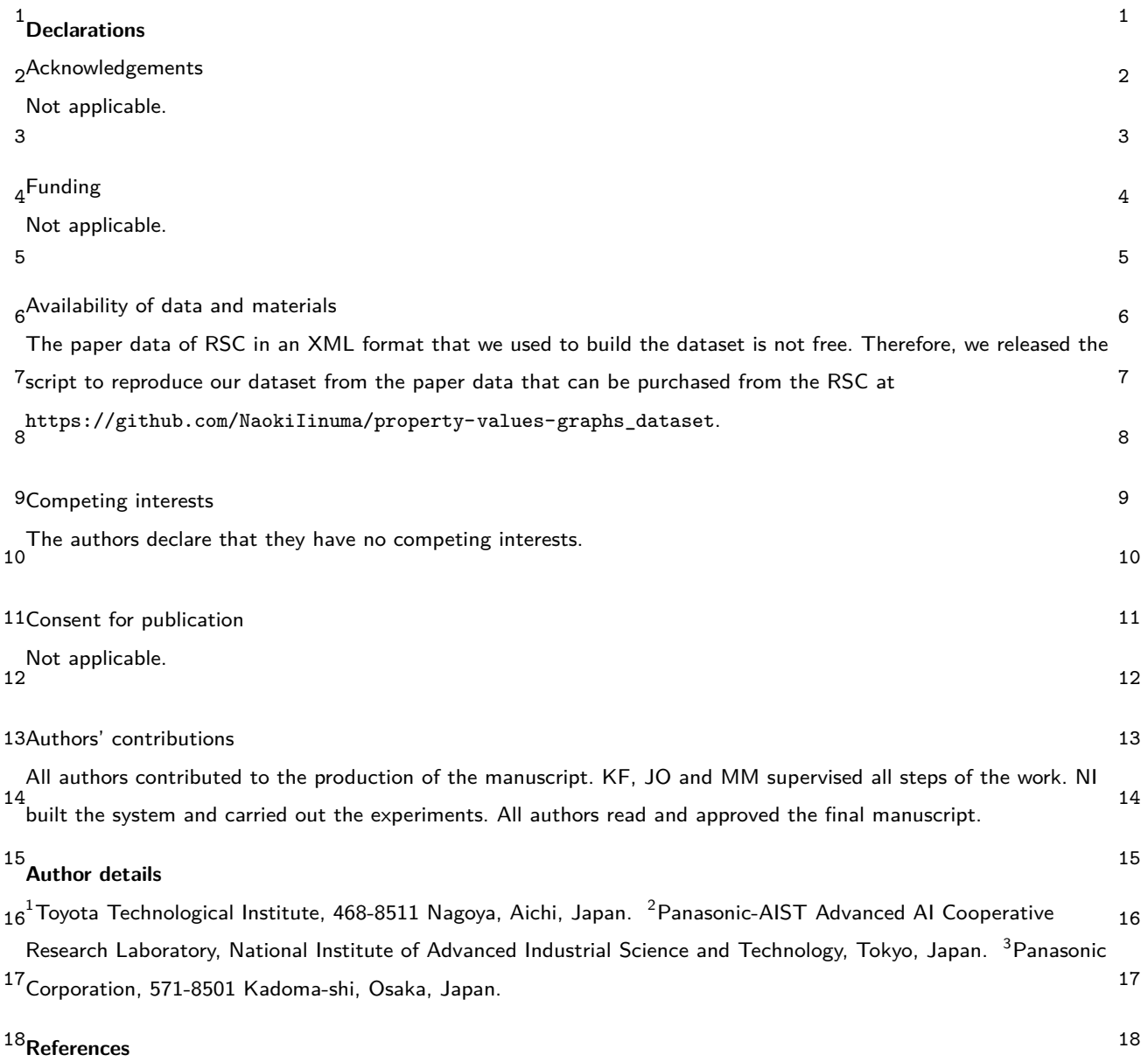

19 1. Kuniyoshi, F., Makino, K., Ozawa, J., Miwa, M.: Annotating and extracting synthesis process of all-solid-state 19 batteries from scientific literature. In: Proceedings of the 12th Language Resources and Evaluation Conference,

20 pp. 1941-1950. European Language Resources Association, Marseille, France (2020). 20 https://www.aclweb.org/anthology/2020.Irec-1.239

21 2. Mysore, S., Jensen, Z., Kim, E., Huang, K., Chang, H.-S., Strubell, E., Flanigan, J., McCallum, A., Olivetti, E.: ${ }^{21}$

22 The materials science procedural text corpus: Annotating materials synthesis procedures with shallow semantic 22 structures. In: Proceedings of the 13th Linguistic Annotation Workshop, pp. 56-64. Association for

23 Computational Linguistics, Florence, Italy (2019). doi:10.18653/v1/W19-4007. 23

24 https://www.aclweb.org/anthology/W19-4007 24

3. Kononova, O., Huo, H., He, T., Rong, Z., Botari, T., Sun, W., Tshitoyan, V., Ceder, G.: Text-mined dataset of

25 inorganic materials synthesis recipes. Scientific Data 6 (2019). doi:10.1038/s41597-019-0224-1 25

26 4. Davila, K., Setlur, S., Doermann, D., Bhargava, U.K., Govindaraju, V.: Chart mining: A survey of methods for 26 automated chart analysis. IEEE Transactions on Pattern Analysis and Machine Intelligence, 1-1 (2020).

27 doi:10.1109/TPAMI.2020.2992028 27

5. Kahou, S., Atkinson, A., Michalski, V., Kádár, Á., Trischler, A., Bengio, Y.: Figureqa: An annotated figure 28 dataset for visual reasoning. ArXiv abs/1710.07300 (2018)

29 6. Siegel, N., Horvitz, Z., Levin, R., Divvala, S., Farhadi, A.: Figureseer: Parsing result-figures in research papers. 29 In: European Conference on Computer Vision (ECCV) (2016)

30 7. Swain, M.C., Cole, J.M.: Chemdataextractor: A toolkit for automated extraction of chemical information from ${ }^{30}$

31 the scientific literature. Journal of Chemical Information and Modeling (2016). doi:10.1021/acs.jcim.6b00207 31

8. Kim, D., Ramesh, B.P., Yu, H.: Automatic figure classification in bioscience literature. Journal of Biomedical

32 Informatics 44(5), 848-858 (2011). doi:10.1016/j.jbi.2011.05.003 32

33 9. Mukaddem, K.T., Beard, E.J., Yildirim, B., Cole, J.: Imagedataextractor: A tool to extract and quantify data 33 from microscopy images. Journal of chemical information and modeling (2020) 
${ }^{1}$ 10. Tsutsui, S., Crandall, D.J.: A data driven approach for compound figure separation using convolutional neural 1

2 networks 01, 533-540 (2017). doi:10.1109/ICDAR.2017.93

11. Wang, G., Xu, H., Lu, L., Zhao, H.: One-step synthesis of mesoporous mno2/carbon sphere composites for

3 asymmetric electrochemical capacitors. J. Mater. Chem. A 3, 1127-1132 (2015). doi:10.1039/C4TA03096F 3

412. He, K., Zhang, X., Ren, S., Sun, J.: Deep residual learning for image recognition, 770-778 (2016). doi:10.1109/CVPR.2016.90

513. Tan, M., Le, Q.: EfficientNet: Rethinking model scaling for convolutional neural networks 97, 6105-6114 5 (2019)

614. Kim, E.,Huang, K., Tomala, A., Matthews, S., Strubell, E Saunders, A, McCallum, A., Olivetti, E.:

7 Machine-learned and codified synthesis parameters of oxide materials. Scientific Data (2017). 7 doi: $10.1038 /$ sdata. 2017.127

${ }^{8}$ 15. Li, L., Jamieson, K., Rostamizadeh, A., Gonina, E., Ben-tzur, J., Hardt, M., Recht, B., Talwalkar, A.: A system ${ }^{8}$

9 for massively parallel hyperparameter tuning 2, 230-246 (2020)

16. Akiba, T., Sano, S., Yanase, T., Ohta, T., Koyama, M.: Optuna: A next-generation hyperparameter

10 optimization framework. In: Proceedings of the 25rd ACM SIGKDD International Conference on Knowledge 10 Discovery and Data Mining (2019)

11 17. Kingma, D.P., Ba, J.: Adam: A method for stochastic optimization. In: Bengio, Y., LeCun, Y. (eds.) 3rd

12 International Conference on Learning Representations, ICLR 2015, San Diego, CA, USA, May 7-9, 2015, 12 Conference Track Proceedings (2015). http://arxiv.org/abs/1412.6980

13. Sutskever, I., Martens, J., Dahl, G., Hinton, G.: On the importance of initialization and momentum in deep

14 learning. In: Dasgupta, S., McAllester, D. (eds.) Proceedings of the 30th International Conference on Machine 14 Learning. Proceedings of Machine Learning Research, vol. 28, pp. 1139-1147. PMLR, Atlanta, Georgia, USA

15 (2013). http://proceedings.mlr.press/v28/sutskever13.html

16 19. Wang, P., Wang, H., Chen, X., Liu, Y., Weng, X., Wu, Z.: Novel scr catalyst with superior alkaline resistance 16 performance: enhanced self-protection originated from modifying protonated titanate nanotubes. J. Mater.

17 Chem. A 3, 680-690 (2015). doi:10.1039/C4TA03519D 17

20. Thomas, P., Pei, C., Roy, B., Ghosh, S., Das, S., Banerjee, A., Ben, T., Qiu, S., Roy, S.: Site specific supramolecular heterogeneous catalysis by optically patterned soft oxometalate-porous organic framework

19 (som-pof) hybrid on a chip. J. Mater. Chem. A 3, 1431-1441 (2015). doi:10.1039/C4TA01304B 19

21. Fan, M.-S., Chen, J.-H., Li, C.-T., Cheng, K.-W., Ho, K.-C.: Copper zinc tin sulfide as a catalytic material for

20 counter electrodes in dye-sensitized solar cells. J. Mater. Chem. A 3, 562-569 (2015). 20

21 doi:10.1039/C4TA02319F 21

22. Lin, Z., Liang, C.: Lithium-sulfur batteries: from liquid to solid cells. J. Mater. Chem. A 3, 936-958 (2015).

22 doi:10.1039/C4TA04727C 22

$23^{23 .}$ Chen, H., Jiang, J., Zhao, Y., Zhang, L., Guo, D., Xia, D.: One-pot synthesis of porous nickel cobalt sulphides: 23 tuning the composition for superior pseudocapacitance. J. Mater. Chem. A 3, 428-437 (2015).

24 doi:10.1039/C4TA04420G 24

24. Chen, G., Wang, S., Yi, R., Tan, L., Li, H., Zhou, M., Yan, L., Jiang, Y., Tan, S., Wang, D., Deng, S., Meng, 25

$\mathrm{X}$., Luo, H.: Facile synthesis of hierarchical mos2-carbon microspheres as a robust anode for lithium ion

26 batteries. J. Mater. Chem. A 4, 9653-9660 (2016). doi:10.1039/C6TA03310E 26

25. Zhang, Y., Bao, X., Xiao, M., Tan, H., Tao, Q., Wang, Y., Liu, Y., Yang, R., Zhu, W.: Significantly improved

27 photovoltaic performance of the triangular-spiral tpa(dpp-pn)3 by appending planar phenanthrene units into 27

28 the molecular terminals. J. Mater. Chem. A 3, 886-893 (2015). doi:10.1039/C4TA03688C 28

26. Han, M.-K., Jin, Y.-S., Yu, B.K., Choi, W., You, T.-S., Kim, S.-J.: Sulfur to oxygen substitution in biocuse and

29 its effect on the thermoelectric properties. J. Mater. Chem. A 4, 13859-13865 (2016). 29 doi:10.1039/C6TA04310K

30 doi:10.1039/C6TA04310K 30

27. Dong, X., Xu, Y., Yan, S., Mao, S., Xiong, L., Sun, X.: Towards low-cost, high energy density li2mno3 cathode

31 materials. J. Mater. Chem. A 3, 670-679 (2015). doi:10.1039/C4TA02924K 31

28. Schitco, C., Bazarjani, M.S., Riedel, R., Gurlo, A.: Nh3-assisted synthesis of microporous silicon oxycarbonitride 32 ceramics from preceramic polymers: a combined $\mathrm{n} 2$ and $\mathrm{co} 2$ adsorption and small angle x-ray scattering study. 32

33 J. Mater. Chem. A 3, 805-818 (2015). doi:10.1039/C4TA04233F 33 
${ }^{1}$ 29. Wang, Z., Liang, M., Tan, Y., Ouyang, L., Sun, Z., Xue, S.: Organic dyes containing dithieno[2,3-d:2 ,3 -d ]thieno[3,2-b:3,2 -b ]dipyrrole core for efficient dye-sensitized solar cells. J. Mater. Chem. 2 A 3, 4865-4874 (2015). doi:10.1039/C4TA06705C

330. Li, Q., Zheng, F., Huang, Y., Zhang, X., Wu, Q., Fu, D., Zhang, J., Yin, J., Wang, H.: Surfactants assisted 3 synthesis of nano-lifepo4/c composite as cathode materials for lithium-ion batteries. J. Mater. Chem. A 3 , 2025-2035 (2015). doi:10.1039/C4TA03293D

531. Mosconi, E., Umari, P., De Angelis, F.: Electronic and optical properties of mixed sn-pb organohalide 5 perovskites: a first principles investigation. J. Mater. Chem. A 3, 9208-9215 (2015). doi:10.1039/C4TA06230B

32. Han, X., Ye, Y., Lam, F., Pu, J., Jiang, F.: Hydrogen-bonding-induced assembly of aligned cellulose nanofibers

7 into ultrastrong and tough bulk materials. J. Mater. Chem. A 7, 27023-27031 (2019). 7 doi:10.1039/C9TA11118B

8

Figures

9

10 Figure 1: Example of classification process of graphs 10

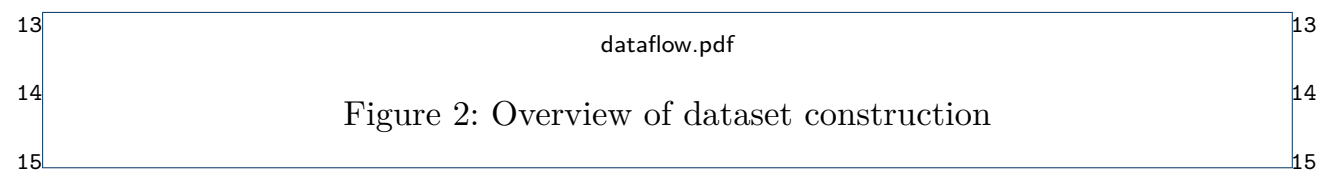

16

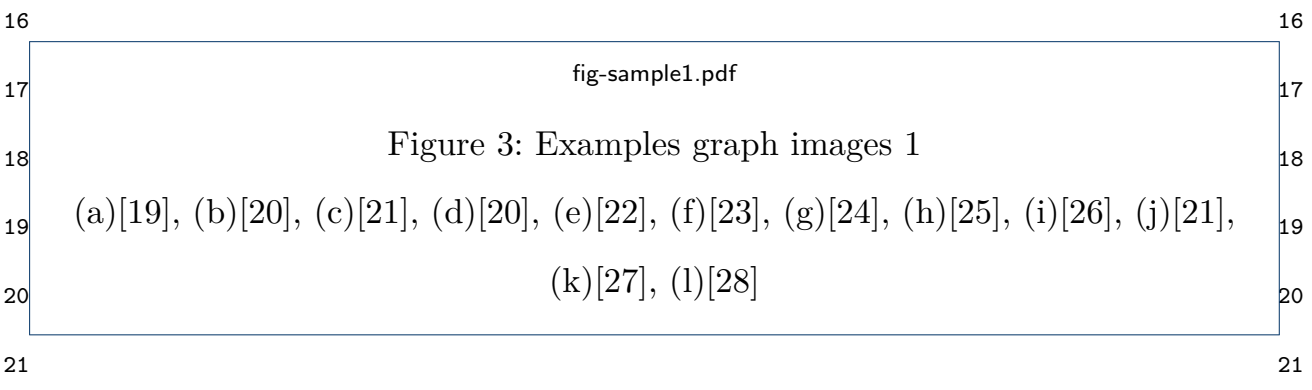

fig-sample2.pdf
23
24

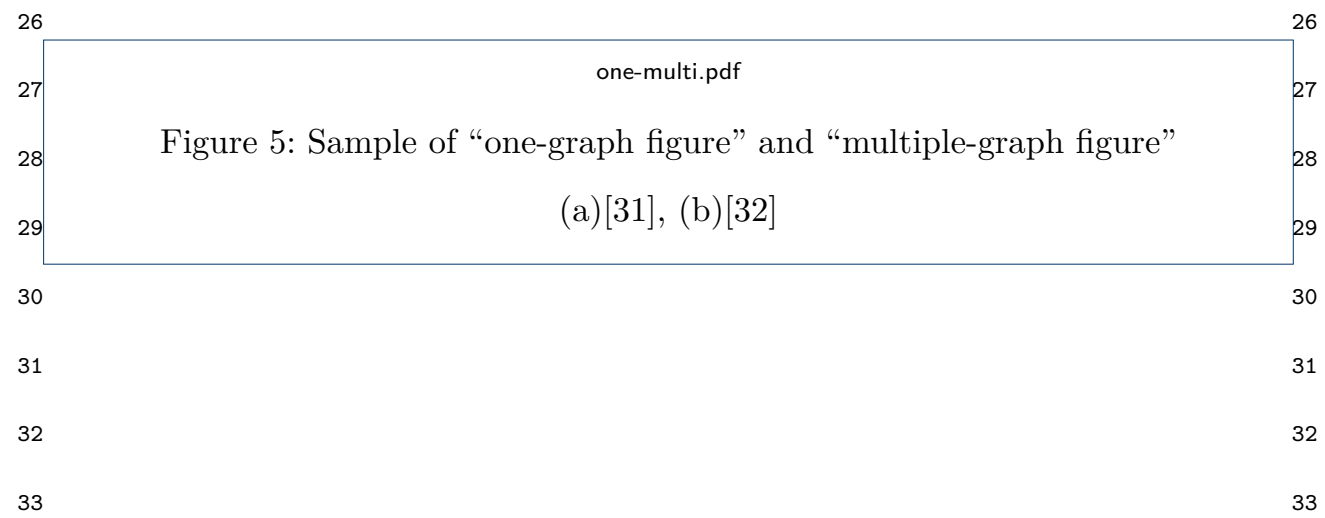


26 \begin{tabular}{lll}
27 & \multicolumn{1}{|c|}{ MatCNN-CM.pdf } \\
28 & Figure 8: Confusion matrix of gold labels (vertical axis) vs. Mat-CNN predictions & 28 \\
29 & (horizontal axis) & 29 \\
2 & 26 & 30
\end{tabular} 


\section{Classify figures}

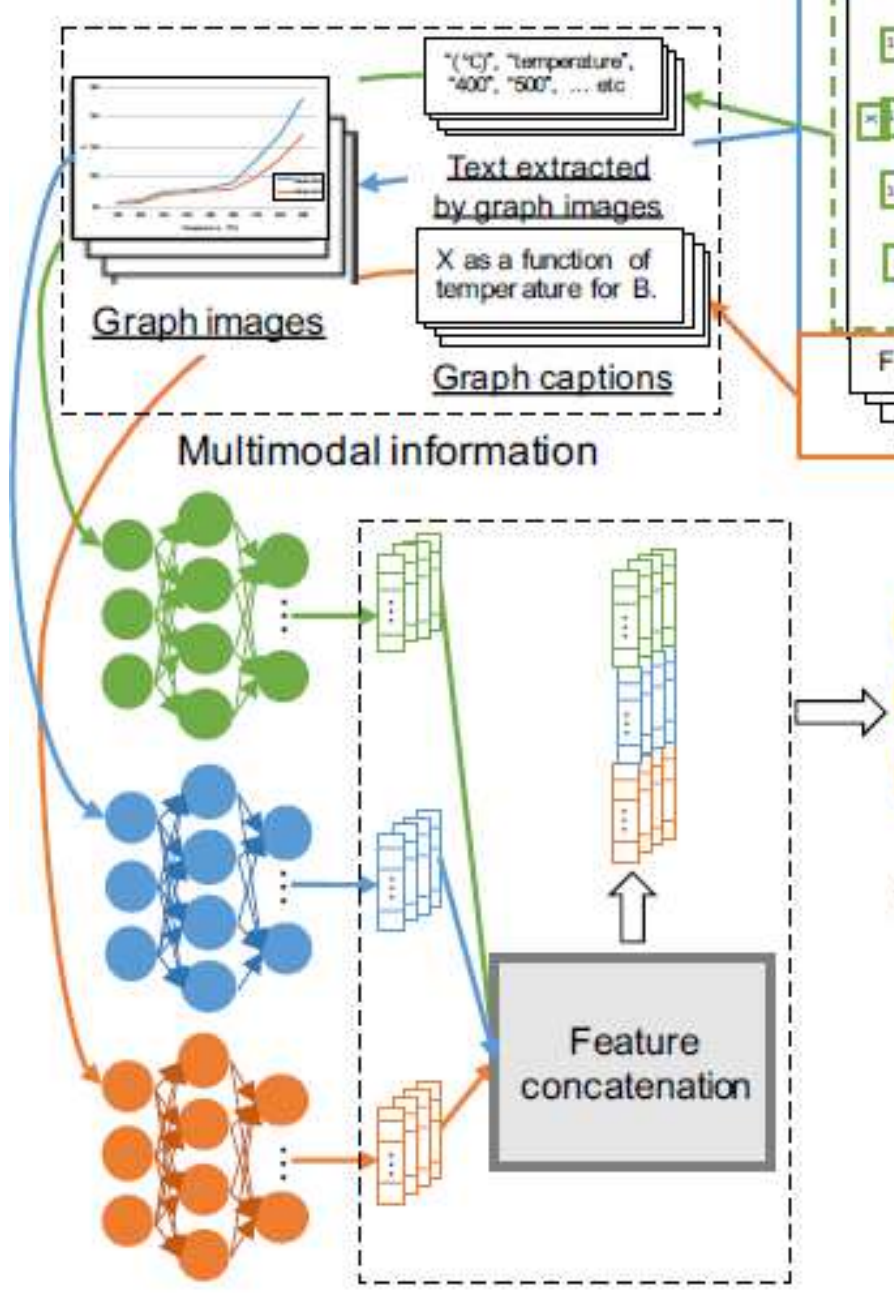

NN feature extractors
Feature vectors

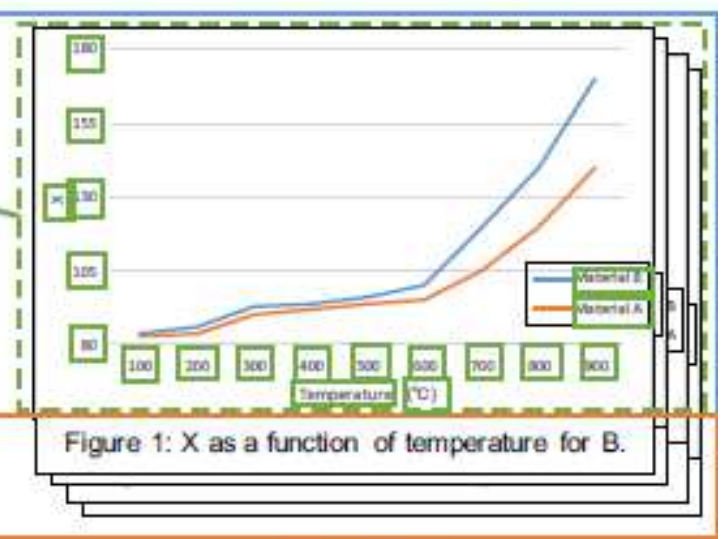

Graphs

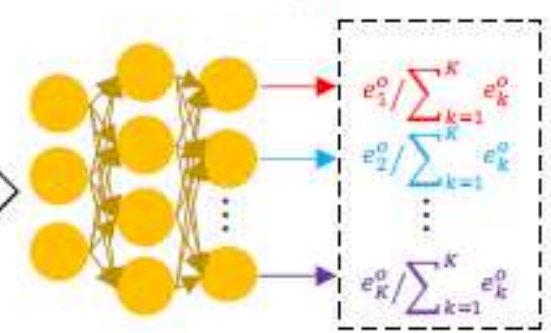

NN Classifier SoftMax

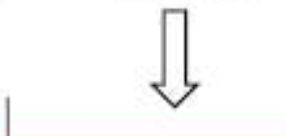

Temperature

Time

:

\section{Energy}

Probabilities

Figure 1

Example of classification process of graphs

\section{Construct a dataset}

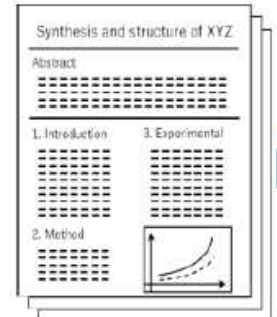

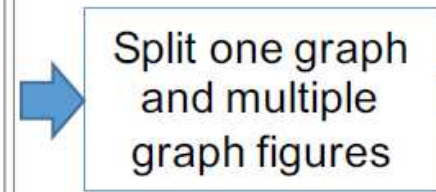

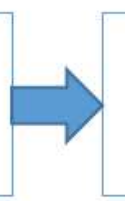
Annotate one graph figures by crowdsourcing
Integrate labels

by the majority voting

XML papers

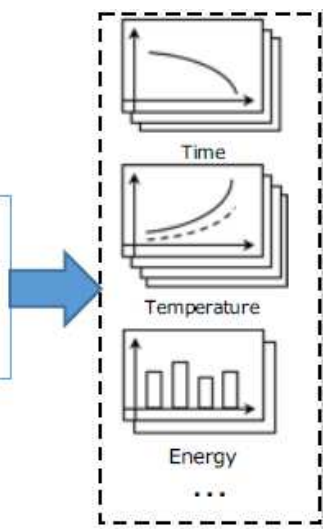

Dataset 
Figure 2

Overview of dataset construction

(a)

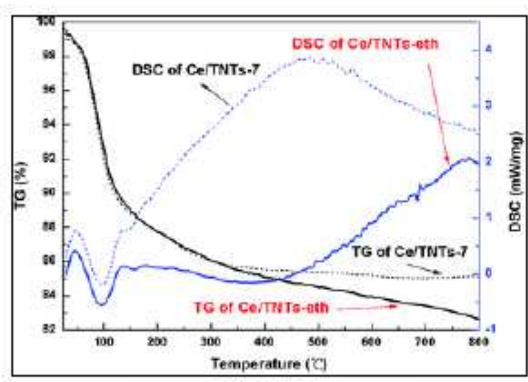

Temperature

(d)

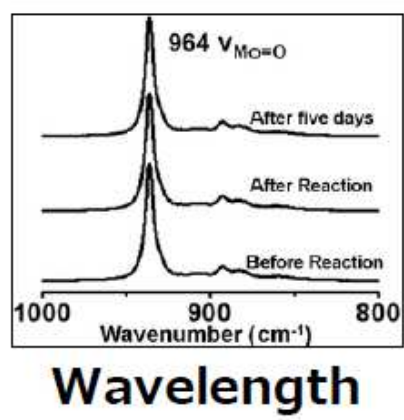

(g)

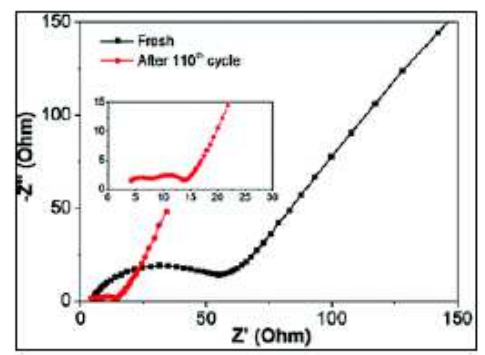

Ohm

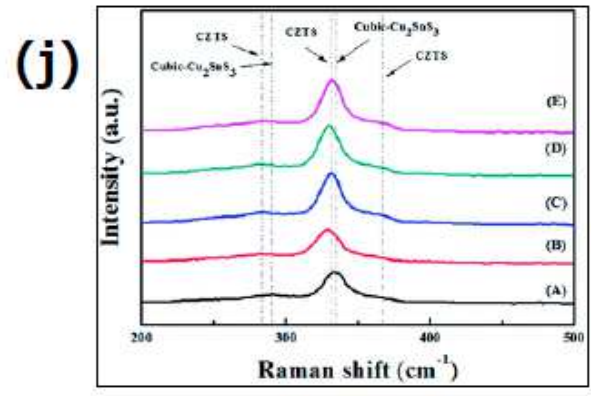

Raman shift (b)

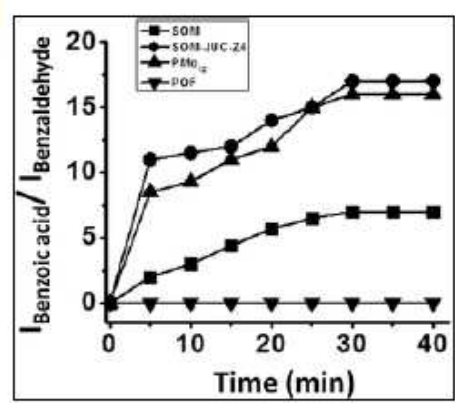

Time

(e)

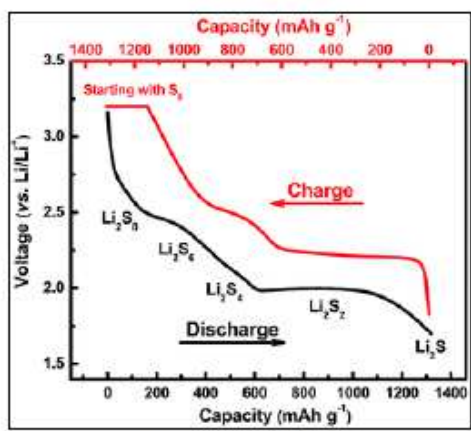

Capacity

(h)

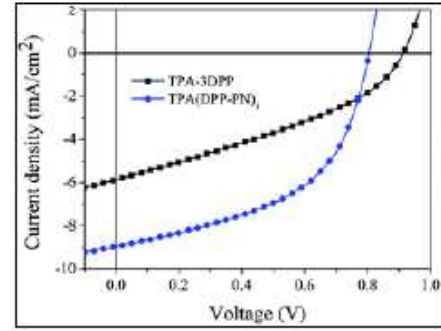

Voltage

(k)

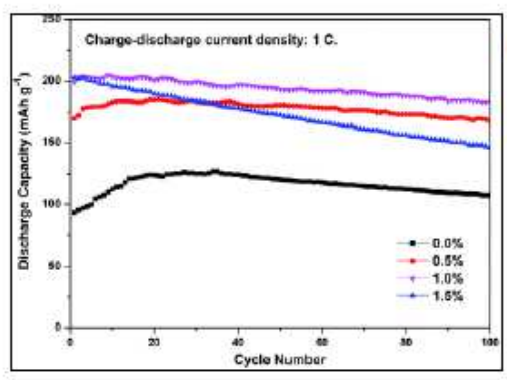

Cycle (c)

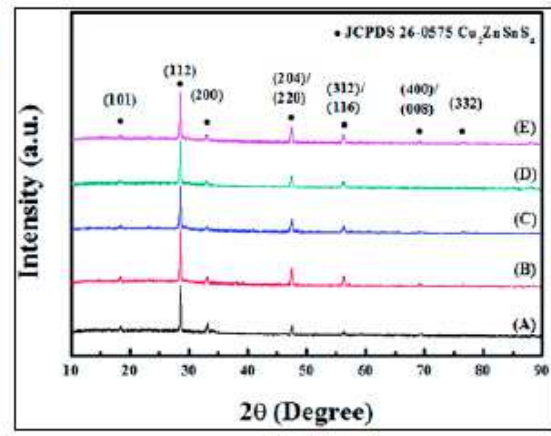

Angre

(f)

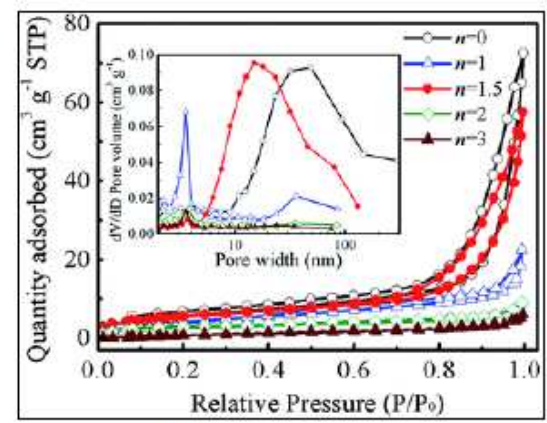

Pressure

(i)

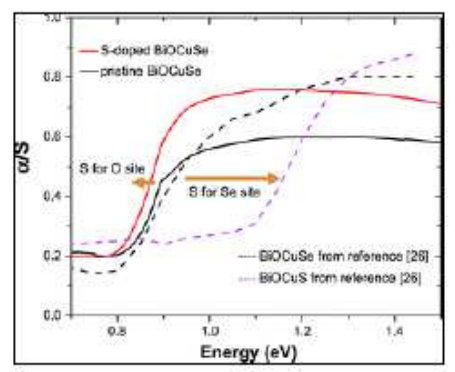

Energy

(I)

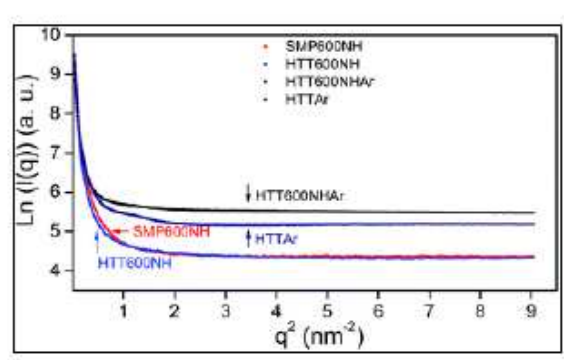

Other

Figure 3

Examples graph images 1 (a)[19], (b)[20], (c)[21], (d)[20], (e)[22], (f)[23], (g)[24], (h)[25], (i)[26], (j)[21], (k) [27], (I)[28] 
(a)
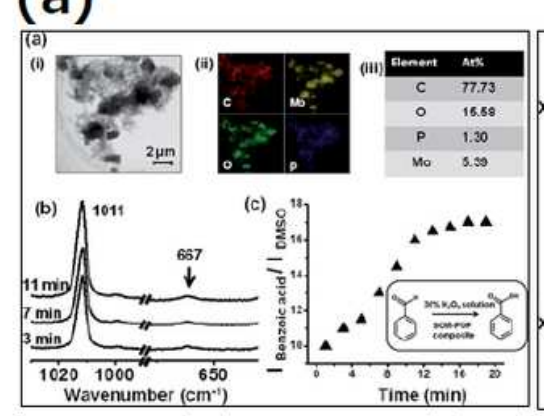

(b)
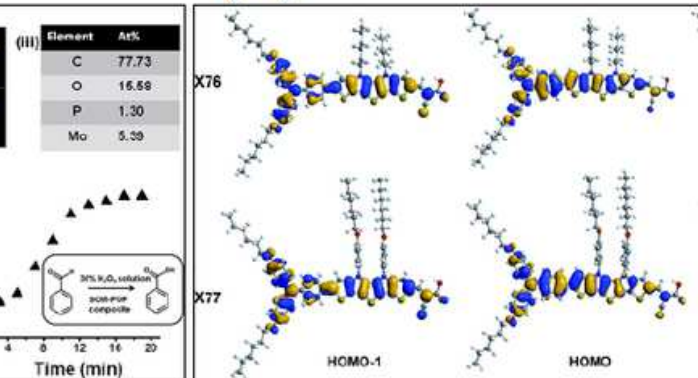

(c)

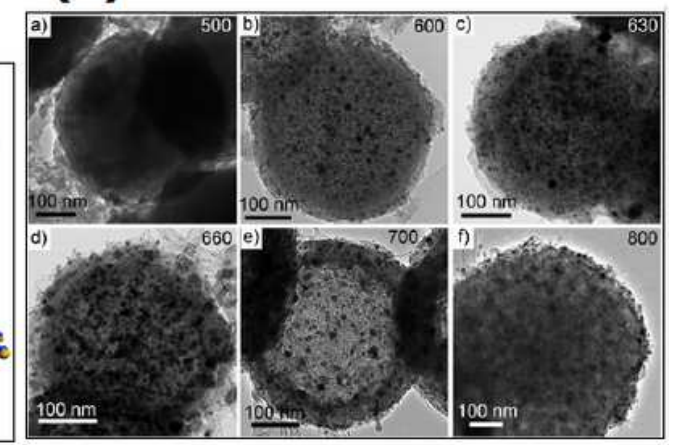

Not Target

Figure 4

Example graph images 2 (a)[20], (b)[29], (c)[30]

(a)

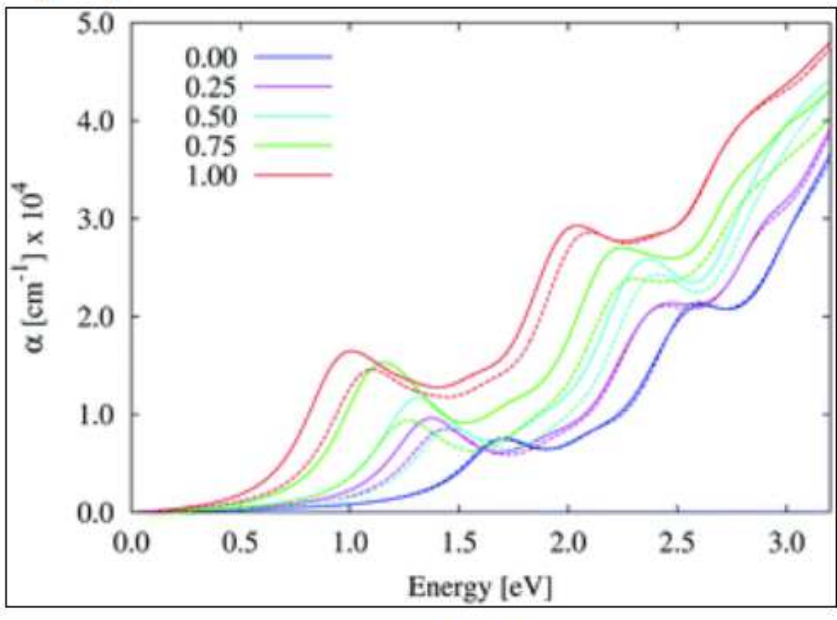

one graph figure (b)

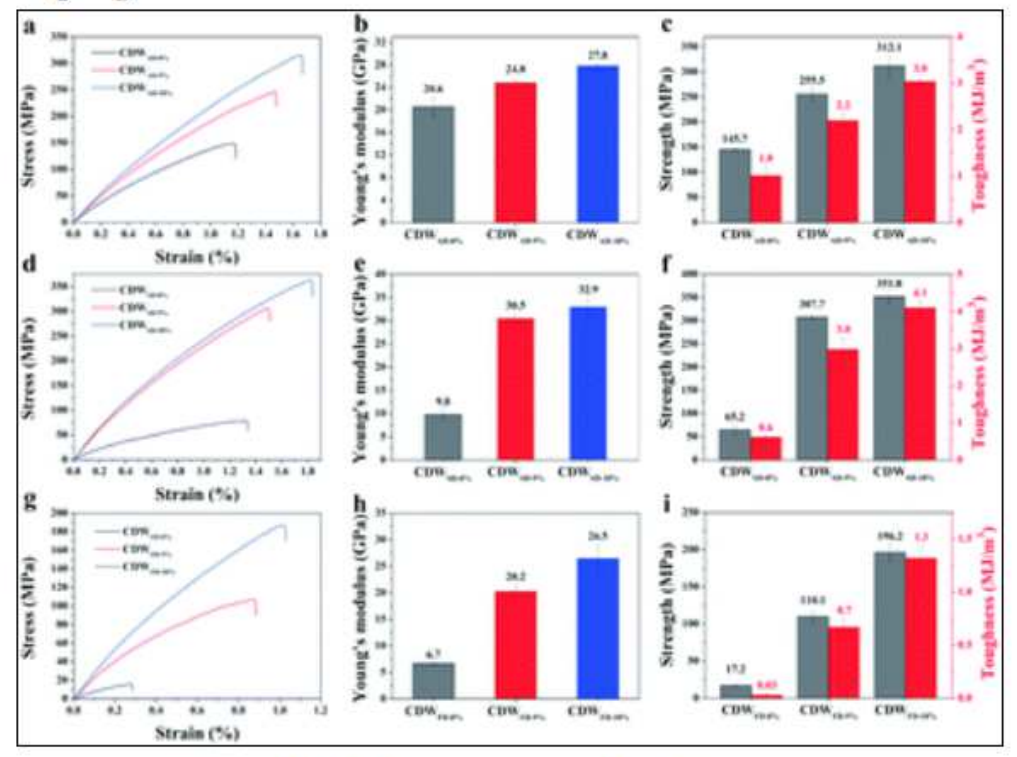

multiple graph figure

Figure 5

Sample of "one-graph figure" and "multiple-graph figure" (a)[31], (b)[32] 


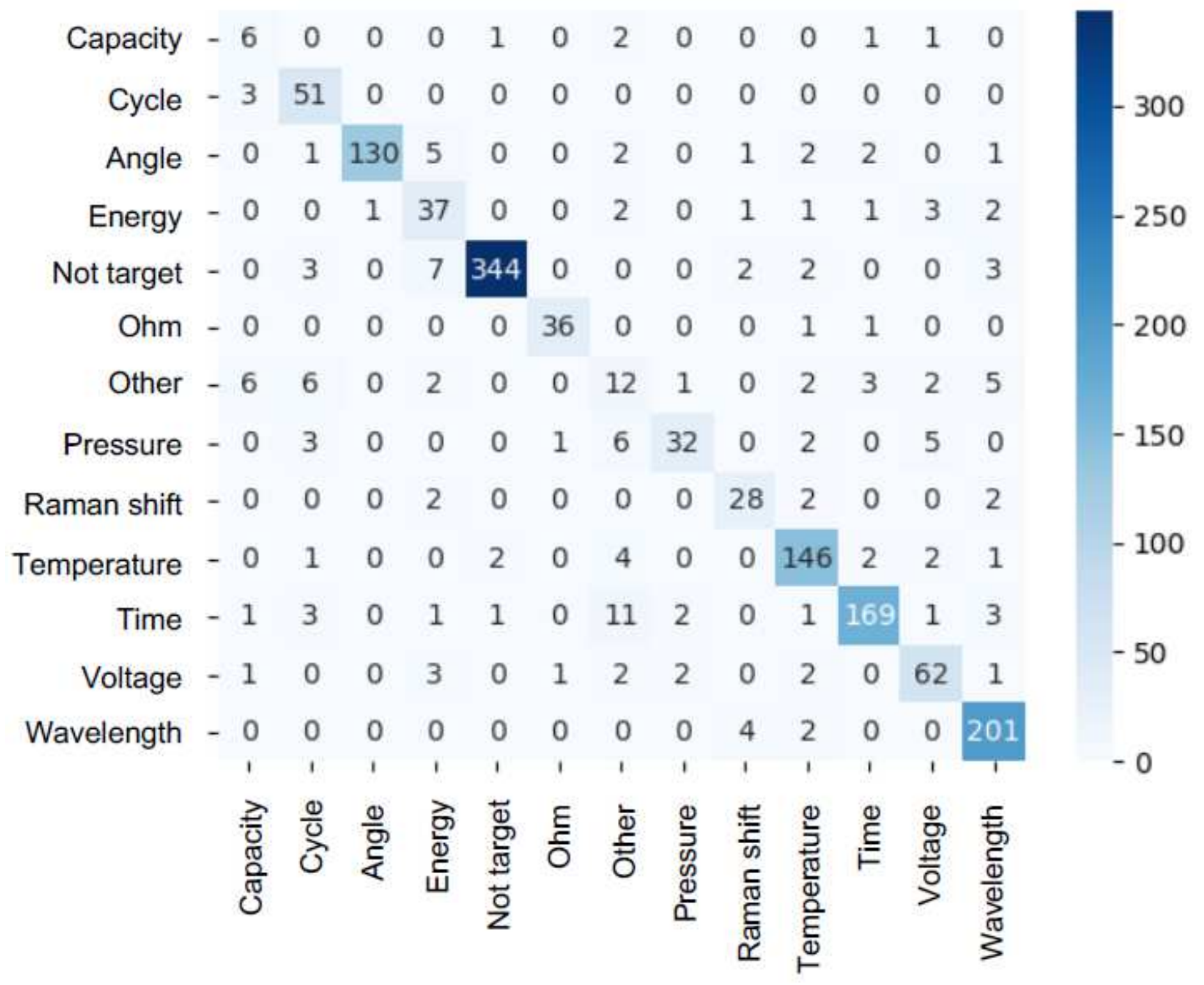

Figure 6

Confusion matrix of gold labels (vertical axis) vs. EfficientNet predictions (horizontal axis) 


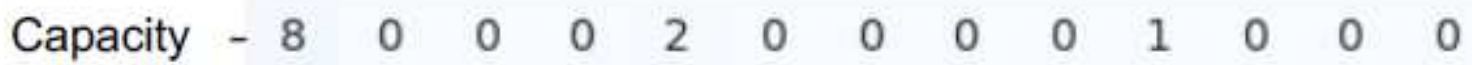
$\begin{array}{llllllllllllll}\text { Cycle - } & 1 & 45 & 0 & 0 & 3 & 2 & 1 & 0 & 0 & 0 & 2 & 0 & 0\end{array}$

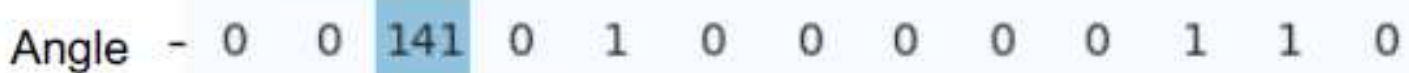
Energy - $0 \begin{array}{llllllllllll}0 & 3 & 41 & 2 & 0 & 2 & 0 & 0 & 0 & 0 & 0 & 0\end{array}$ Not target - $\begin{array}{lllllllllllll}0 & 2 & 1 & 4 & 347 & 0 & 3 & 0 & 0 & 0 & 1 & 3 & 0\end{array}$ Ohm - $\begin{array}{lllllllllllll}0 & 2 & 3 & 0 & 2 & 29 & 1 & 0 & 0 & 0 & 0 & 1 & 0\end{array}$ Other - $\begin{array}{lllllllllllll}0 & 2 & 6 & 0 & 7 & 0 & 19 & 1 & 0 & 2 & 1 & 1 & 0\end{array}$ Pressure - $0 \begin{array}{lllllllllllll} & 0 & 0 & 0 & 0 & 0 & 0 & 48 & 0 & 0 & 1 & 0 & 0\end{array}$ $\begin{array}{llllllllllllll}\text { Raman shift - } & 0 & 0 & 0 & 0 & 0 & 0 & 0 & 0 & 33 & 0 & 0 & 0 & 1\end{array}$ Temperature $-\begin{array}{llllllllllllll}0 & 2 & 1 & 0 & 2 & 1 & 3 & 0 & 0 & 147 & 2 & 0 & 0\end{array}$ Time - $0 \begin{array}{lllllllllllll} & 1 & 0 & 0 & 3 & 0 & 2 & 0 & 0 & 1 & 184 & 2 & 0\end{array}$ Voltage - $\begin{array}{ccccccccccccc}0 & 1 & 1 & 0 & 6 & 0 & 0 & 0 & 0 & 1 & 3 & 62 & 0\end{array}$ $\begin{array}{lllllllllllllll}\text { Wavelength - } 0 & 0 & 0 & 0 & 2 & 0 & 0 & 0 & 0 & 0 & 0 & 1 & 204 & -0\end{array}$

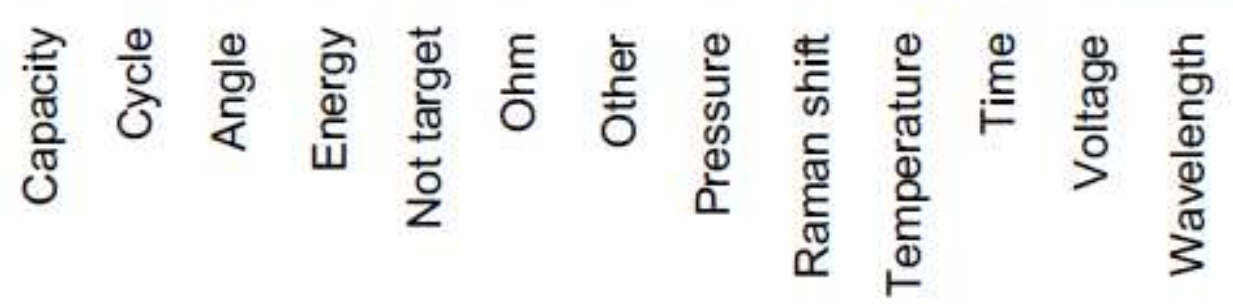

Figure 7

Confusion matrix of gold labels (vertical axis) vs. BoW predictions (horizontal axis) 


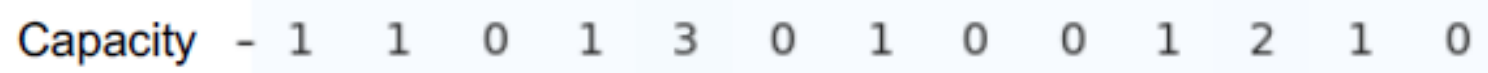

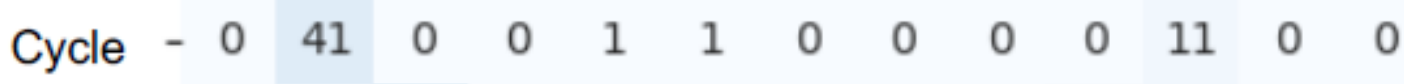
Angle - $0 \begin{array}{lllllllllllll} & 138 & 0 & 1 & 0 & 0 & 0 & 0 & 4 & 0 & 0 & 0\end{array}$ Energy - $0 \begin{array}{lllllllllllll}0 & 0 & 37 & 3 & 0 & 1 & 0 & 0 & 2 & 0 & 0 & 5\end{array}$ Not target - $\begin{array}{lllllllllllll}0 & 0 & 3 & 3 & 343 & 1 & 2 & 1 & 0 & 0 & 6 & 1 & 1\end{array}$ Ohm - $0 \begin{array}{lllllllllllll}0 & 0 & 0 & 0 & 38 & 0 & 0 & 0 & 0 & 0 & 0 & 0\end{array}$ $\begin{array}{llllllllllllll}\text { Other }- & 0 & 2 & 1 & 1 & 6 & 0 & 6 & 2 & 0 & 8 & 9 & 2 & 2\end{array}$ Pressure - $0 \begin{array}{lllllllllllll}0 & 2 & 0 & 0 & 0 & 0 & 1 & 39 & 0 & 5 & 2 & 0 & 0\end{array}$ $\begin{array}{llllllllllllll}\text { Raman shift }- & 0 & 0 & 0 & 0 & 0 & 0 & 0 & 0 & 30 & 0 & 0 & 0 & 4\end{array}$

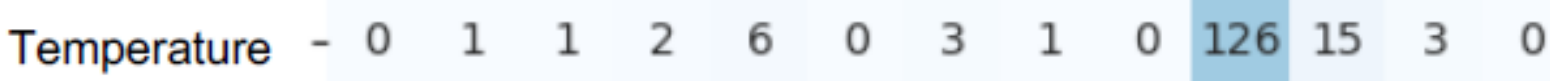
Time - $\begin{array}{lllllllllllll}0 & 7 & 0 & 0 & 10 & 1 & 4 & 1 & 0 & 4 & 155 & 7 & 4\end{array}$

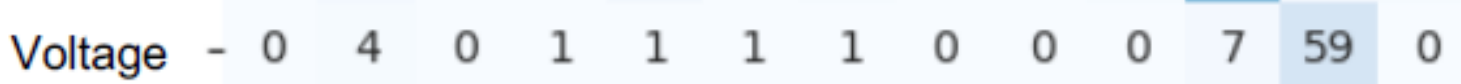
$\begin{array}{lllllllllllllll}\text { Wavelength }-0 & 0 & 2 & 4 & 4 & 0 & 0 & 0 & 10 & 1 & 3 & 0 & 183 & -0\end{array}$

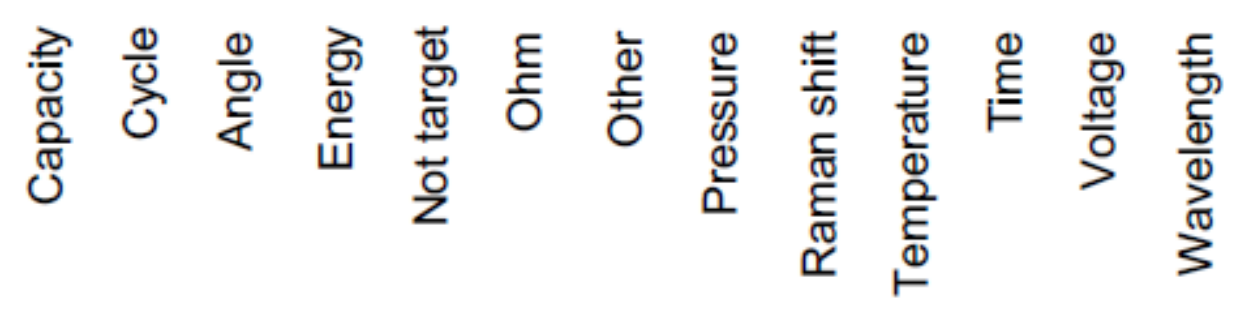

Figure 8

Confusion matrix of gold labels (vertical axis) vs. Mat-CNN predictions (horizontal axis) 